


\section{Un análisis histórico-filosófico acerca de la medición del tiempo en las cronologías bíblicas}

\section{A historical-philosophical analysis about the measurement of time in biblical chronologies}

Carlo Del Razo Canuto Escuela Nacional de Antropología e Historia, México

Resumen: Este artículo analiza los distintos desafíos a los que se enfrentaron los cronólogos bíblicos al tratar de calcular la fecha de La Creación durante el periodo histórico conocido como la Antigüedad Tardía hasta el siglo xviI, desafíos tales como: ¿Cuándo fue el momento exacto de La Creación? ¿O cómo podemos calcular ese tiempo? Desde un análisis histórico-filosófico se exponen las dificultades conceptuales, epistémicas, metodológicas y cognitivas a las que se enfrentaron los cronólogos en su intento por establecer una escala temporal que les permitiera fechar de manera absoluta toda la historia humana incluyendo el origen del Mundo. Las preguntas filosóficas esbozadas aquí son acerca del rol, la representación, la evaluación y las limitaciones epistémicas de las escalas de tiempo.

Palabras clave: ciencias históricas, medición científica, filosofía de la medición, escalas de tiempo, medición del tiempo.

Abstract: This article analyzes the different challenges that biblical chronologists faced when trying to calculate the date of Creation during the historical period known as Late Antiquity until the 17th century, challenges such as: When was the exact moment of The Creation? Or how can we calculate that time? From a historical-philosophical analy- 
sis are exposed the conceptual, epistemic, methodological and cognitive difficulties faced by chronologists in their attempt to establish a time scale that would allow them to date absolutely all human history including the origin of the World. The philosophical questions sketched here are about the role, representation, evaluation and epistemic limitations of time scales.

Keywords: Historical Sciencies, Scientific measurement, Philosophy of measurement, Time scales, Measurement of time.

Recibido: 28 de junio de 2018 Aceptado: 29 de octubre de 2018

Any enquiry into the past which does not reckon with the dimension of time is obviously nonsense.

Piggott

\section{Introducción}

T a historia temprana de las ciencias históricas nacidas entre los - siglos XVII y XIX ha sido representada mediante el socavamiento de los principios de la naturaleza establecidos a partir de la interpretación literal de la Biblia, incluyendo la cronología bíblica que establece que el origen del Mundo fue en el año 4004 a. C. Stephen J. Gould (1993: 333) ha sido justo en señalar que los libros de texto no alcanzan a mostrar completamente lo que significó el establecimiento de una cronología completa para toda la historia humana. Éste es el mismo señalamiento que planteo aquí. La historiografía de la cosmología, la geología, la arqueología, así como la paleontología y la biología evolutiva, han minimizado la importancia que tuvo la tradición de cronólogos bíblicos en su afán por establecer con precisión la edad de la Creación. Si bien muchas de 
estas representaciones describen con claridad cuáles fueron las condiciones y consecuencias que impulsaron el crecimiento del conocimiento histórico, y hallaron en la ruptura del cálculo bíblico uno de los componentes más importantes, el elemento historiográfico que no está presente es la explicación de los artilugios utilizados en la constitución de un nuevo sistema de creencias naturalizado sobre el origen del Mundo, incluyendo la descripción de los elementos de las cronologías bíblicas que fueron superados, así como aquellos que permanecieron sin modificación en la investigación de fenómenos del pasado.

Sorprende todavía más que muchos historiadores y filósofos de la ciencia utilicen esta aparente ruptura con las ideas pre-cientificas para medir el progreso de la ciencia. Thomas Kuhn (1977) opinaba acertadamente que este tipo de creencias que aprende un estudiante como parte de su especialidad crea profundas raíces en las comunidades científicas. Es por ello que Kuhn advirtió en contra de basar una filosofía de la ciencia en los libros de texto ya que éstos tergiversan el proceso y la evolución histórica de la investigación y la teorización (vid. Kuhn, 1996). Desde Kuhn han pasado generaciones de filósofos que han señalado lo limitado de las representaciones históricas.

Philip Kitcher relaciona a este tipo de representaciones con un tipo de ceguera: "The failures of understanding, however, rest on the neglect of history. Epistemology without history proves blind in this instance, because it deprives us of any satisfying explanation for the knowledge we have" (Kitcher, 2011: 515). Kitcher utiliza el caso de las matemáticas para mostrar cómo la falta de consideración de la historia no ofrece una explicación satisfactoria del conocimiento; su naturalismo pragmático está inspirado en el reconocimiento de que las prácticas científicas tienen una larga historia, y que el estudio de ésta puede ofrecer una forma de explicar los problemas filosóficos de la ciencia. 
Otro elemento que está ausente en las representaciones históricas tradicionales y en la reflexión filosófica es el tema de la medición cientifica. La medición ha sido uno de los principales recursos "por el cual la ciencia ha obtenido información del mundo empírico" (Guillaumin, 2012: 57). Guillaumin han señalado que a pesar de la importancia que tiene la medición para el desarrollo y crecimiento del conocimiento científico, existe un abandono sistemático por la gran mayoría de los estudios de la filosofía de la ciencia (vid. Guillaumin, 2012: 59). Para el caso de las ciencias históricas el diagnóstico es similar, y el tema de la medición del Tiempo es ilustrativo. El Tiempo es aquello en lo que los procesos o eventos históricos están incrustados. Sin embargo, y a pesar de la importancia que ha tenido la cronología en el desarrollo y crecimiento del conocimiento histórico, también es notable la ausencia de estudios histórico-filosóficos que pretendan entender los aspectos conceptuales, epistémicos, metodológicos y cognitivos relacionados con las prácticas de la medición del tiempo en estas disciplinas.

Annick Lesne (2017) ha argumentado que la representación del Tiempo es un modelado en cual se establece por medio de la integración de escalas epistémicas y escalas intrínsecas. De acuerdo con esto, las escalas epistémicas debemos entenderlas como escalas mediante las cuales se observan y describen los sistemas naturales; es decir, aquellas que parten de la apariencia observable y mesurable. Las escalas temporales establecidas por evidencia documental o por medio del registro fósil son ejemplos de escalas epistémicas. Por otro lado, las escalas intrínsecas son aquellas que se establecen a partir de los tiempos característicos de los sistemas naturales y son independientes de la observación y de la descripción. La tasa de enfriamiento de un cuerpo incandescente, los ciclos de los cuerpos celestes o la formación de un perfil estratigráfico son ejemplos de una escala intrínseca. Esta distinción es fundamental en este artículo ya que me va permitir diferenciar los problemas epistémicos 
de los problemas sistémicos, además de trazar los limites de cada uno de ellos.

Este trabajo tiene el objetivo de mostrar que la medición del Tiempo en la tradición de las cronologías bíblicas involucró un conjunto de elementos que se fueron desarrollando, modificando y reconstituyendo con la finalidad de establecer una escala temporal absoluta que abarcara toda la historia humana. El carácter histórico del estudio está enfocado en mostrar cuáles fueron las ideas, las prácticas y los métodos empleados para medir y establecer dicha escala en torno a tres principales artilugios: el uso de genealogías, de documentos históricos (principalmente la Biblia), así como distintos calendarios civiles y astronómicos. Acerca del carácter filosófico, el énfasis principal está relacionado con la explicación de tres elementos utilizados para establecer una escala epistémica que sirviera de base para calcular la edad de la humanidad, a saber: criterios epistemológicos, reglas metodológicas y recursos cognitivos.

Hay dos razones por las cuales este estudio es importante. La primera es que trata de mostrar que el principal problema para fechar el inicio del Mundo en la cronología bíblica fue sostener que la Tierra y la Humanidad fueron producto de un mismo acto de creación. Esto quiere decir que para calcular ese momento, los cronólogos bíblicos trataron de establecer una escala temporal absoluta por medio del mismo tipo de evidencia: la documental. En cambio, en el siglo XVII hay una evidente ruptura metodológica al tratar de establecer una escala temporal relativa por medio del estudio directo de los objetos de la naturaleza o evidencia física. El cambio metodológico fue sustantivo para postular por primera vez que la Tierra tenía una historia geológica e independiente de la historia humana. La segunda razón es que la fecha establecida por las cronologías bíblicas fue el único cálculo aceptado por la comunidad de eruditos, anticuarios y filósofos naturales hasta la segunda mitad del siglo XIX cuando se habían establecido las condiciones de 
posibilidad para postular que el Mundo era más antiguo respecto al cálculo bíblico; lo cuál dio paso a la investigación del tiempo profundo en geología, la prehistoria en arqueología y la evolución de las especies en la biología y en la paleontología.

\section{Principios físicos. El origen del mundo y la estructura del tiempo}

En la concepción judeo-cristiana el cálculo para saber de la edad del Mundo estuvo ligado durante muchos siglos al estudio de la antigüedad humana. Esta concepción dio por hecho que para conocer la edad de la Tierra bastaba saber cuántos años tenía la humanidad en ella. En el primer capítulo del Génesis se afirma que Adán - el primer hombre- había sido formado después de cinco días de incansable creación por la mano de Dios. Se acepta que la Tierra siempre ha sido un mundo humano, lo que significa que tal como es en el presente es tal y como fue creada; se da por hecho que lo escrito dentro del Génesis es el relato histórico más antiguo que registra los primeros orígenes del Mundo (Rudwick, 2014: 12) y se acepta que lo dicho en la Biblia es literalmente verdadero, dado que no hay algún otro tipo de evidencia que indicara que el origen había sido de otra forma.

Por lo tanto, la noción del Tiempo dentro de la tradición de los cronólogos bíblicos debemos considerarla bajo algunas ideas subyacentes a ella. Pascal Richet señala que dentro de la estructura del Antiguo y Nuevo Testamento existe la idea de una historia lineal (Richet, 2007: 34) o una flecha del tiempo como la llamaré aquí. Stephen J. Gould utiliza esta metáfora aludiendo a la idea de que "cada momento ocupa una posición específica en una serie temporal, y todos los momentos, considerados en la secuencia adecuada, determinan una sucesión de eventos enlazados que se mueven en una dirección" (Gould, 1987: 29). Esta idea da por hecho la 
existencia de un marco temporal irreversible y delimitado por un principio y un final donde existen sucesiones de acontecimientos irrepetibles y únicos (Richet, 2007: 29).

En el pensamiento judeo-cristiano esta idea implicó la creencia de que el Tiempo es quien dota de una estructura rígida y determinada a los eventos, lo cual permite saber cuáles son anteriores, contemporáneos o posteriores a otros. Entonces la noción de estructura rígida debe ser entendida como el conjunto de relaciones temporales establecidas entre eventos, donde cada uno de ellos queda ubicado dentro de una escala temporal sin la posibilidad de ser desplazable; no ser desplazable es una característica intrínseca de un evento. Al quedar establecido como principio físico que la estructura del tiempo es lineal, el estudio de la historia humana requirió asentarse dentro de una escala temporal fija e inmutable desde los primeros tiempos y a lo largo de la historia. A esta escala le llamo escala temporal epistémica, la cual se fue estableciendo mediante una larga tradición de estudios genealógicos basados inicialmente en el Génesis y posteriormente en el Nuevo Testamento. El Génesis proporcionó los primeros datos genealógicos sobre Adán y nueve de sus descendientes del linaje de Seth, incluyendo las cifras para la edad de cada patriarca hebreo cuando engendró a su primogénito hasta el Diluvio narrado por Noé (vid. Nothaft, 2011: 191). El Nuevo Testamento, por su lado, proporcionó una visión escatológica impuesta por la idea del Juicio Final, lo que significaría el Fin de los Tiempos (vid. Barr, 1984, 1999, 2005; Wilcox, 1987; Wallraff, 2007).

\section{El problema métrico. El mundo comienza con la creación pero no se sabe cuándo}

La idea central en la tradición de cronologías bíblicas es la creencia de que la Humanidad, la Tierra y el Tiempo fueron creados en el 
mismo momento en que Dios culminó la Creación, y fue aceptada de manera casi incuestionable por los estudiosos de la cronología desde la antigüedad. Al aceptarla como verdadera, los cronólogos tuvieron que lidiar con dos problemas fundamentales que apuntaron directamente a un problema métrico: ¿Cuándo fue el momento exacto de la Creación? ¿O cómo podemos calcular ese tiempo?

En la primera epístola al lector de los Annales veteris testamenti, a prima mundi origine deducti (1650), el obispo James Ussher afirma la idea de una Creación que incluye la formación de los Hombres y el Mundo como parte de un mismo evento. Sin embargo, enfatiza que sus predecesores no podían establecer un cálculo confiable sobre el origen del Mundo. Para Ussher fue evidente que el desconocimiento puntual de las Sagradas Escrituras fue uno de los obstáculos principales que dificultó el cálculo para fechar el momento de la Creación. La segunda razón es que tuvieron que pasar muchos años para que el conocimiento astronómico detrás de los distintos tipos de calendarios mediante los cuales se establecieron distintas escalas de tiempo fuera más exacto: "Anyone can do this who is well versed in the knowledge of sacred and secular history, of astronomical calculations and of the old Hebrew calendar. If he should apply himself to these difficult studies, it is not impossible for him to determine not only the number of years but even the days from the creation of the world" (Ussher, 2003: 10). El estado de la cuestión tal y como Ussher lo evalúa en el siglo Xvir es ilustrador, ya que permite poner en contexto el problema dentro de la tradición de cronólogos bíblicos.

Existe dos importantes desarrollos intelectuales que contribuyeron a la elaboración de una escala de tiempo absoluta como la propuesta por Ussher. Por un lado, los siglos XVI y XVII fueron testigos de una nueva metodología que empleó técnicas críticas para evaluar la fiabilidad de las fuentes documentales antiguas. En segundo lugar, se desarrolló un nuevo sistema de fechamiento 
basado en observaciones astronómicas que hizo posible integrar eventos de diferentes sistemas cronológicos en un sólo sistema: el calendario Juliano. A continuación expongo cómo se fueron integrando históricamente los distintos elementos que constituyeron la tradición de cronologías bíblicas hasta la formulación del cálculo de 4004 años a. C. en las investigaciones de Ussher.

\section{Herramientas de cálculo en las cronologías bíblicas durante la antigüedad tardía}

\section{Genealogias biblicas}

Retomando la noción de flecha del tiempo, la tradición de cronólogos bíblicos estableció como primer recurso metodológico el uso de las genealogías hebreas para hacer cronologías. Desde El Génesis, hasta los libros de Los Hechos, los principales acontecimientos históricos estuvieron conectados por medio de las sucesiones genealógicas de los principales patriarcas hebreos. Si nos remontamos brevemente a los primeros siglos de nuestra Era, veremos que por esa época comenzaron aparecer importantes libros de cronología bíblica basada en sucesiones genealógicas. Flavius Josephus (37-100 d. C.) en su Jewish Antiquieties (vid. Momigliano, 1978; Wilcox, 1987; Feldman, 1998; Rajak, 2002), o bien el Seder Olam Rabba, escrito por el rabino Rose ben Halafta -alrededor del año de 160 d.C.- (Véase Grafton y Williams: 2006; Richet: 2007; Wentraun: 2011; Rudwick: 2014), sentaron las primeras bases para la elaboración de las cronologías bíblicas por medio de la recopilación de datos que describían la fundación de ciudades, festivales, rituales, leyes, costumbres, etc.

Una de las estrategias metodológicas más importantes en estos trabajos pioneros fue el establecimiento de genealogías que permi- 
tieran conocer cuántos años habían vivido algunos de los personajes más importantes del Antiguo Testamento. Bajo la idea de 'quién engendró a quién y cuándo', estos autores trataron de establecer una escala temporal que permitiera medir de manera absoluta cuántos años habían pasado desde que Dios creó a Adán. En el caso de Josephus, el procedimiento para establecer dicha escala dependía en gran medida en el establecimiento de líneas genealógicas de parentesco y de las edades de los patriarcas registradas en la Biblia. Estos últimos datos eran computados por medio de una operación aritmética simple como la suma, y luego eran comparados con algunas fechas disponibles registradas en otros documentos históricos hebreos o griegos. Para Josephus las Escrituras ofrecían una continuidad única que comenzaba con Adán y procedía por generaciones a través de los principales patriarcas y reyes de Israel, cuyos regencias podían sincronizarse con los eventos de la historia clásica griega (Wilcox, 1987: 104). Sin embargo, Josephus le otorga una mayor antigüedad y mayor legitimidad a las Escrituras sobre los documentos clásicos griegos en cuanto al origen del Mundo, menciona que: "the Jews have been here all the time and, unlike the Greeks, who have a lot of different and contradictory books, the Jews have one precise and unified history, one single narrative that goes back to the creation of the world about five thousand years before" (Josephus apud. Barr, 1999: 380).

Distintos autores trabajaron a lo largo de los primeros siglos bajo esta regla intentando perfeccionar las primeras cronologías documentales basadas en la lectura de la Biblia. Julius Africanus (160-232 d. C.), realizó cronologías documentales desde una perspectiva escatológica. Esta perspectiva se basó en el supuesto de que los hechos proféticos escritos en el Antiguo Testamento habían sido confirmados históricamente. Grafton y Williams (2006: 151) mencionan que la tarea principal de la cronología histórica de Africanus fue dilucidar el futuro por adelantado; para lograr 
dicho objetivo, tenía que recrear un pasado bajo la creencia de que éste debería tener una duración fija y ordenada, similar a lo que he llamado aquí la metáfora de la flecha del tiempo. Por tal motivo, para Africanus fue normal pensar que el resto de los hechos descritos en los libros sagrados le permitirían predecir también el Fin de los Tiempos. Martin Wallraff ha señalado que la idea principal de Africanus era insertar la historia humana en un sistema cronológico aritméticamente coherente que abarcase todas las ramas de la historia, incluidas las tradiciones semíticas y helenísticas. Este sistema estaba apoyado en la convicción cristiana de que la duración de toda la historia humana ascendía a 6000 años, de acuerdo con la idea de los seis días de la Creación, por lo cual cada día equivalía a 1000 años (vid. Wallraff, 2007: XXIX-XXX).

En su Chronographiae (221 d. C.), Africanus utilizó como regla metodológica la comparación de las fechas importantes dentro de la Biblia con las fechas registradas en otros documentos históricos judíos, cristianos y paganos, principalmente fechas establecidas por calendarios astronómicos y civiles. Este tipo de procedimiento se basó en búsqueda sistemática de 1) los principales personajes descritos en el Antiguo Testamento en otros documentos históricos con el objeto de estudiar su parentesco y finalmente reconstruir su genealogía; o bien, 2) la búsqueda sistemática de eventos laicos importantes con el fin de correlacionarlos con los bíblicos. En el primer caso, la existencia de relaciones genealógicas no sólo eran capaces de mostrar quién era el antecesor común, sino también el grado de cercanía temporal que existía entre cada uno de ellos.

En el segundo caso, el procedimiento metodológico basado en la comparación de fuentes documentales le permitió a Africanus correlacionar los eventos importantes narrados en la Biblia con otros tipos de eventos registrados dentro de los calendarios egipcio, persa, griego, caldeo y romano. Por ejemplo, Africanus analizó las fechas de aquellos eventos separados espacialmente cuyas 
temporalidades se cruzaban, y menciona: "I will take one Hebrew event that is simultaneous with an event recorded by Greeks and by sticking to it, while both adding and subtracting, and indicating which Greek or Persian or anyone else synchronized with that Hebrew event, I may perhaps achieve my goal." (Africanus apud. Grafton/Williams, 2006: 141-142). La finalidad de este procedimiento fue ordenar temporalmente un mayor número de eventos históricos registrados en distintos tipos de documentos.

\section{Sincronismo}

El término de sincronismo fue una palabra técnica utilizada en los estudios clásicos griegos sobre cronología documental y sirvió para referirse a la datación o fechamiento de cualquier evento registrado en múltiples sistemas de cómputo cronológico. En la Chronographiae de Africanus, la sincronicidad es una idea metodológica que le permitió establecer valores cruzados temporalmente entre la cronología bíblica y la cronología griega. Por medio de este procedimiento estableció que se puede fechar de manera absoluta uno o más eventos registrados en dos o más líneas cronológicas. Esto se debe a que la sincronización permite calibrar fechas entre distintas líneas cronológicas mediante una misma unidad de medida. $\mathrm{Al}$ igual que Josephus, Africanus desestima los documentos griegos tempranos por parecerle confusos en cuanto al registro de las fechas y solamente acepta como apropiados aquellos que tienen registros bajo una unidad de medida satisfactoria: "The Greek accounts of history are by no means accurate before the beginning of the Olympiads, but thoroughly confused and in total disagreement with one another. But there are many accurate accounts of events after this point, since the Greeks made their records of them every four years, rather than at longer intervals" (Africanus apud. Grafton/Williams, 2006: 142). Africanus menciona que en el An- 
tiguo Testamento los judíos comenzaron a ser enviados a Palestina en el primer año del reinado de Ciro, rey de Persia. Díodoro y otros historiadores fecharon el primer año de reinado de Ciro para la Olimpiada número 55, la cual está registrada para el año 560/59 a. C, por lo cual, el reinado de Ciro y el fin del cautiverio hebreo coinciden (vid. Africanus Libro III, F34; Grafton/Williams, 2006: 149; Wallraff, 2007: 163-169). Wallraff menciona que gracias a Africanus, el sincronismo se convirtió en un medio para mediar entre dos sistemas cronológicos e históricos diferentes (Wallraff, 2007: p. XXII).

Al hablar de la historia temprana Africanus fue muy cauteloso, sin embargo, sostiene que las obras adjudicadas al profeta Moisés son las de mayor antigüedad comparada con cualquier otra obra escrita por algún pagano en cuanto al registro de los primeros tiempos. Cuando se trataba de los orígenes de la humanidad, todos los historiadores helénicos aceptaban como el comienzo del Tiempo la Olimpiada 1. Para Africanus, la incertidumbre de los historiadores griegos sobre la historia profunda (calculada en 4727 años) sólo podía ser reconstruida sobre la base segura de la cronología judía.

For the Jews, deriving their origin from them as descendants of Abraham, having been taught a modest mind, and one such as becomes men, together with the truth by the spirit of Moses, have handed down to us, by their extant Hebrew histories, the number of 5500 years as the period up to the advent of the Word of salvation, that was announced to the world in the time of the sway of the Cæsars (Africanus apud. Georgius Syncellus, Chron: 81, al. 65).

Africanus termina de escribir la Chronographiae en el año $221 \mathrm{~d}$. C., en la cual abarca un periodo de 5723 años. La datación de Cristo, por ejemplo, la establece en el año 5531 AM, 192 años antes de la Olimpiada número 250. 
Sincronización de cronologias por medio de sistemas tabulares

Mediante la sincronización se va establecer una idea de solidez documental. Esta idea se va ir desarrollando durante los siguientes siglos con la búsqueda de un mayor número de fuentes documentales que permitieran establecer valores cruzados mucho más fiables. Tal es el caso del Chronicon (325 d. C) escrito por Eusebio de Cesarea (265-340). Eusebio fue el principal erudito de la antigüedad encargado de sentar las bases para determinar la fecha exacta de la Creación por medio de la sincronización de varias líneas cronológicas. En su Chronicon establece las mismas reglas metodológicas que Africanus, sin embargo, existen algunas diferencias importantes. En el trabajo de Africanus, la principal dificultad que enfrenta la sincronización es que para calibrar con mayor precisión las fechas de eventos históricos se necesita más de una fuente documental. En cambio, Eusebio concibió la idea de que era posible elaborar una cronología histórica a través de los registros de acontecimientos narrados en textos no bíblicos -i.e., historias de Asiria, Egipto y Roma-, aunque la principal escala temporal siguiera siendo fundamentada en el calculo bíblico. Eusebio menciona que:

[...] the history of the Hebrew patriarchs as revealed in the Bible. And thus we might establish how long before the life-giving revelation [of Christ] Moses and the Hebrew prophets who succeeded him lived and what they, filled with the divine spirit, said before [the time of Christ]. In this fashion it might be possible to recognize easily when the braves of each nation appeared [compared with] when the celebrated Hebrew prophets lived and, one by one, who all their leaders were (Cesarea, 2008).

La contribución de Eusebio se vio reflejada en una gran cantidad de tablas cronológicas por medio de las cuales pudo estable- 
cer un ajuste temporal entre distintas líneas paralelas de datos: "I will convert all the material collected about all these folk into chronological tables. Including, from the beginning, who from each nation ruled as king and for how long, I will put these [facts] into separate [chronological tables] together with the number of years involved. In this way, if we need to know who ruled and for how long [that information] will be easily and quickly accessible" (Cesarea, 2008).

Las tablas fueron elaboradas mediante columnas verticales en las cuales se enlistaban datos como los nombres de los reyes y sus dinastías, algunos eventos importantes y sus fechas calculadas por medio de calendarios astronómicos y civiles. El número de columnas dependía del número de países que había en determinado momento. La columna izquierda contiene la continuidad y sucesión de los imperios asirio, persa y romano (vid. también Wilcox, 1987: 105-106). En estas columnas se iban incorporando fechas ordenadas, primero lo más antiguo después lo más reciente. Este ingenioso recurso de sincronización le permitió a Eusebio trazar líneas horizontales entre columnas que tuvieran un evento fechado el mismo año; la simultaneidad de dos o más sucesos le permitía establecer valores mucho más precisos para así ajustar el resto de las tablas. Este método para fechar los acontecimientos del pasado prevaleció durante muchos siglos en la tradición de cronólogos bíblicos, pues el formato tabular como fue concebido obligaba a cualquier historiador que deseara escribir historia antigua a ser preciso con cualquier dato que se integrara a las tablas ya que de ello dependían las subsecuentes sincronizaciones y el evitar errores. Wilcox menciona que el intento de sincronizar la secuencia espiritual de la historia hebrea con la secuencia política de la historia helenística requirió siglos en aclarase, sin embargo, el uso práctico de las tablas fue fecundo durante la Edad Media hasta que en los 
siglos XVI y XVII se establecieron los cimientos de la historia secular que abolió tal distinción (Wilcox, 1987: 107).

Respecto al cálculo de la historia profunda el sistema tabular había mostrado el mismo problema epistemológico que el sistema de Africanus: en una cronología documental, el cómputo de ańos de la historia temprana no siempre se puede determinar con precisión ya que muchas de las fuentes no registran los primeros ańos relacionados con el nacimiento de los reinos. La respuesta a este problema es que para Eusebio no existe una forma, que no sea mediante la autoridad de Dios, para conocer realmente la edad de la Tierra. Esta idea es sumamente importante ya que la precisión final que se requiere para calcular la edad de la Creación sólo puede estar en manos de Dios; en otras palabras, Dios no solamente es el creador del Tiempo, sino que también es el único que tiene el intelecto para calcularlo: "for it is not possible to know unerringly the chronology of the entire world, not from the Greeks, not from the barbarians, not from other [peoples], not even the from the Hebrews (Cesarea, 2008).

En busca de una escala de tiempo absoluto. Cronologías bíblicas en los siglos XVI y XVII

La cronología bíblica cultivada por Josephus, Africanus y Eusebio se convirtió en una disciplina durante los siglo XVI y XVII (vid. Schneer, 1954; Grafton, 1983; Nothaft, 2011; Buchwald/Feingold, 2013). Grafton menciona que gran parte del trabajo de los cronólogos de la antigüedad sirvió principalmente como una herramienta para instruir a los conversos en el trabajo histórico, así como una herramienta para refutar las insinuaciones de los paganos sobre lo novedoso del Judaísmo. A pesar de ello, la cronología bíblica en la Antigüedad Tardía no fue una disciplina en sí misma ya que no fue practicada por un cuerpo bien definido de eruditos 
y tampoco contaba con un vocabulario técnico bien desarrollado, ni con objetivos claros o una metodología digna de ser alabada. Lo más importante es que no había ninguna noción clara de lo que constituía el método adecuado en el análisis de las fuentes antiguas (vid. Grafton, 1983: 3). Grafton menciona que: "Between 1450 and 1600 this situation changed. The revival of classical education made the correction and interpretation of literary texts a more urgent task than it had been for a millennium. Humanists began to argue that the art of correcting and explicating texts must become a university discipline" (Grafton, 1983: 3).

El redescubrimiento de fuentes documentales implicó la revisión de puntos técnicos dentro de las cronologías de la historia occidental cristiana, pues ésta comenzaba a ser de interés para un amplio público de estudiosos (vid. Buchwald/Feingold: 2013). El descubrimiento del Nuevo Mundo y el contacto renovado con el Oriente presentaron problemas intelectuales sin precedentes; tenían que conciliar los cálculos de la historia con nuevos hechos que no podían acomodar en el marco temporal de los 6000 años. En las cronologías de Eusebio era relativamente fácil de integrar los eventos conocidos por la tradición judeo-cristiana, pero el sistema no podía integrar las cronologías de aquellas culturas ampliamente separadas en el tiempo. Fue necesario el desarrollo de una cronología absoluta que no se limitase a los calendarios y las épocas tradicionales, sino una cronología que debía extenderse a todas las culturas humanas posibles (vid. Grafton, 1975; Wilcox, 1987). No debemos perder de vista que los nuevos cronólogos heredan el mismo problema que no fue resuelto por los fundadores de las cronologías bíblicas: establecer con precisión las fechas de eventos históricamente importantes, incluyendo el momento de la Creación.

De acuerdo con James Barr, hasta el siglo xviI, la Biblia seguía siendo el libro que proporcionaba una secuencia cronológica bastante clara de la Creación y de toda la historia posterior, pero 
habían tres principales problemas métricos: el primero de ellos es que dentro de la misma Biblia había dos o más referencias cronológicas de un mismo evento con fechas diferentes; el segundo es que para el Siglo xvir ya existían tres tradiciones textuales de la Biblia que diferían por periodos de tiempo bastante largos, especialmente desde Adán hasta Noé, y desde Noé hasta Abraham (Barr menciona que el período que va desde la Creación hasta el Diluvio, el texto hebreo tradicional dio un período de 1,656 años; pero el texto griego, la Septuaginta, dio para el mismo período una cifra de 2,242 años. En el Siglo xvir el texto hebreo de los samaritanos -que se suponía el más antiguo de todos- tenía fechas en dirección opuesta teniendo una cifra más baja de 1307 años para el mismo período); el tercer problema métrico, se debió a que, en cierto sentido, no puede haber una cronología bíblica sin salir de la Biblia. Barr menciona que la cronología hebrea al final se desvanece: funciona bastante bien desde La Creación hasta el final de los reinos hebreos, pero después de eso tiene sólo vagos consejos; por ejemplo, sobre el imperio persa, aunque menciona varios de sus emperadores, nadie puede asegurar sólo por la Biblia cuántos reyes persas hubo o cuánto tiempo existió el imperio (vid. Barr, 1999: 382). Estos fueron los principales problemas que trataron de resolver los cronólogos bíblicos modernos.

\section{Análisis y critica de fuentes}

Con la aparición de nuevas culturas de oriente, y las que ya se conocían del Nuevo Mundo y Asia, fue necesario desarrollar un nuevo sistema cronológico documental que abarcara todas las cronologías de imperios y pueblos conocidos. Muchos historiadores comenzaron a tener problemas con la integración del tiempo sagrado y secular en una misma escala temporal, algunos otros comenzaron a preguntarse sobre la veracidad de las fuentes y de los 
medios tradicionales de validación epistémica. El problema de que ninguna fuente era consciente de todos los acontecimientos del pasado planteo nuevos desafíos en materia de validación. Incluso surgió la duda acerca de la originalidad de aquellos documentos que habían integrado las cronologías antiguas. ¿Qué fuentes eran confiables y cuáles no? Wilcox menciona que:

The issues of comprehensiveness and validation were closely connected. As writers in the late sixteenth century felt more acutely the problems of integrating a complex variety of events drawn from independent sources and as they worked more actively to create a history that was truly universal in scope, they saw clearly the need for a new method of validation. They had to establish a system for deciding in principle which sources were accurate and which were not. Only thus could they create a history that would include all possible events, those now part of the historical record and others that might come to light in the future in sources not yet discovered (Wilcox, 1987: 190).

Hacia finales del siglo XVI y principios del XVII, Joseph-Juste Scaliger (1540-1609), recuperando el trabajo de Eusebio, consideró que una cronología debería hacer uso de todas las fuentes disponibles, especialmente de los documentos seculares, con el fin de esclarecer algunos episodios del Antiguo Testamento que permanecían oscuros. Scaliger fue considerado como uno de los eruditos más importantes en Europa en esta época, pues a él se deben dos de los tratados sobre cronología bíblica más populares por aquella época: De emendatione Temporum, de 1583, y el Thesaurus Temporum, de 1606. En ambos trabajos buscó formalizar los estudios cronológicos y establecerlos como una ciencia. Las razones fueron varias, una de ellas se debe al clima intelectual en Europa durante los siglos XVI y XVII. Este período marca el comienzo de la edad moderna, lo que trae la voluntad de cuestionar la autoridad bíbli- 
ca. Grafton menciona que cronólogos antes de Scaliger vieron el campo de la cronología desde una perspectiva religiosa (vid. Grafton, 1983). Sin embargo, los nuevos cronólogos no se restringieron exclusivamente a los límites marcados por la perspectiva de los estudios tradicionales, sino que estos estaban dispuestos a enfocarse en nuevas investigaciones: "All of them were writing chronology not in order to recover an unknown past but in order to prove points in polemics that lay outside the field of chronology -for the most part, points in theology or in the comparative history of religion" (Grafton, 1975: 170).

Durante su juventud, Scaliger se dedicó a la crítica textual de autores latinos por medio del redescubrimiento de documentos antiguos que habían aparecido. El trabajo filológico de estos textos había corregido algunos pasajes que anteriormente parecían traducciones inconsecuentes y arbitrarias. Scaliger criticó severamente las prácticas filológicas superficiales que presentaban la traducción de textos sin un análisis basado en las pruebas presentadas por los mismos manuscritos (vid. Blake, 1940; Wilcox, 1987; De Jonge, 1996). El redescubrimiento de la crónica de Eusebio, cuyo original griego había desaparecido por completo excepto por algunas citas en autores bizantinos, fue consecuencia del estudio de textos latinos basados en la crítica de fuentes documentales. Tras años de estudio, en 1601 llegó al autor del siglo Ix, George Syncellus, cuya crónica resultó tener más fragmentos de Eusebio de los que se conocían en ese momento (vid. Grafton, 1975: 170).

El trabajo de Scaliger consistió en cotejar nuevamente los eventos más importantes que se habían registrado desde la Creación hasta la Edad Media, por un lado, a través de la comparación de fuentes documentales usadas por Eusebio y, por otro lado, por medio de tablas astronómicas de cincuenta calendarios astronómicos diferentes que equiparó con los principios astronómicos descubiertos recientemente por Copérnico y Tycho Brahe (Blake, 1940: 88). 
Con una rigurosa crítica de las fuentes identificó y corrigió los errores de cálculo que habían cometido sus predecesores, lo que le permitió ajustar los conteos dentro de las historias de diversas culturas. Scaliger menciona que:

Thus far we have not only described the years and the civil dates of all nations insofar as we have been able to rescue them from the perpetual silence of oblivion, but also have prepared the way for the ready comparison and coordination of these systems with the Julian and civil calendar of our day. There remains the task of bringing home, so to speak, by means of some methodical guide, a chronology which wanders far over all the earth, and strays like some errant stranger back to the beginnings of earliest antiquity... (Scaliger apud. Blake, 1940: 88).

\section{Characterem e indictio}

Hay dos elementos básicos en el método de Scaliger que fueron de gran relevancia para el desarrollo de un sistema de fechamiento absoluto mediante las cronologías bíblicas (vid. Grafton, 1975: 162; Wilcox, 1987: 197). El primero de ellos, como ya se mencionó, es la implementación de un nuevo estándar de precisión por medio de la crítica de fuentes; el segundo, un método para tratar cada fecha como un punto real y único de la escala temporal. Scaliger estableció con la mayor precisión posible los ańos reales y las respectivas divisiones de la escala de los calendarios anteriores; analizó y clasificó cuidadosamente los datos de sus predecesores e insistió que parte del problema radicaba en el desconocimiento de las fechas encontradas en las fuentes existentes (vid. Wilcox, 1987: 197). Estos elementos le permitieron el agrupamiento y sincronización de los antiguos estudios cronológicos, principalmente los de Eusebio, sobre la base del calendario Juliano como veremos en seguida. 
Para diseñar un sistema de cronología absoluta (chronicon absolutissimum) que fuera completo y autónomo, Scaliger separó a la cronología de sus funciones morales y religiosas, y la convirtió en una disciplina puramente numérica. El número producido en su sistema indicaba sólo en qué momento ocurrió un evento o serie de eventos específicos y no cuál era su significado moral (Wilcox, 1987: 197). Para ello desarrolló un método para identificar cada fecha como un punto separado y distinguible de cualquier otra fecha en el calendario Juliano. Scaliger había señalado que sus antecesores no habían logrado crear una cronología universal porque no aplicaron un characaterem o notación real del año registrado en los distintos sistemas cronológicos. "I find two reasons why they did not succeed in finding out the truth. First, because they did not know the civil chronologies of the ancients, the forms, states, and kinds of years and months. Second, because they did not apply a 'character' [characterem] and notation to the year which they had conceived of" (Grafton, 1975: 162). Wilcox menciona que, debido a la falta de este characterem en los registros documentales los cronólogos, no podían incorporar la información en un sistema de fechamiento que intrínsecamente comprendía todos los eventos de la historia humana (vid. Wilcox, 1987: 198).

Al seguir el trabajo de Dionysius Exiguus (470-544 d. C.), Scaliger ofreció un medio para crear ese characterem dentro de las cronologías. Dionysius buscó un medio para identificar la fecha real de la Pascua. Descubrió que si podía calcular la fase lunar en el día de la semana de la Pascua podría identificar el año exacto en un ciclo de 532 ańos (vid. Wilcox, 1987: 198). Este ciclo de 532 ańos fue calculado mediante la multiplicación de un ciclo solar de 28 años (el cual es usado para computar en qué día de la semana caería un día determinado del año) por un ciclo lunar de 19 años (ideado para regular un calendario lunar-solar y para predecir aproximadamente cuándo caerían las lunas nuevas durante un año 
civil basado en el Sol); el ciclo fue suficiente para el calendario de la Pascua, pero no para que Scaliger estableciera un sistema cronológico universal y absoluto (vid. Grafton, 1975: 162). Scaliger tomó el ciclo de 532 años de Dionysius, pero además incluyó el ciclo basado en la indictio: "Inasmuch as we need to designate each epoch with a character [characterem], the order of times [ratio temporum] will require not only the lunar and solar cycles but also the indictions [indictio]" (Scaliger apud. Wilcox, 1987: 198). La indictio fue un ciclo de quince años establecido por el emperador romano Diocles durante el siglo III en el cual se debía realizar un censo fiscal. Además de esto, Wilcox menciona que: "A historian trying to use the indiction [indictio] as a means of dating a charter would need additional information, such as the name of the reigning monarch, or a reference that would permit location in the solar cycle, such as the day of the week" (Wilcox, 1987: 199). Fue así que Scaliger creó un ciclo lo suficientemente largo que fuera compatible con el calendario Juliano al multiplicar el ciclo establecido por Dionysius por el ciclo de la indictio; es decir, $532(19 \times 28)$ x 15, dando una escala de 7980 ańos.

El establecimiento del calendario Juliano en la cronología de Scaliger sirvió para fechar eventos cuyo characterem fuera conocido. Por ejemplo, si se conociera en qué día del año ocurrió un eclipse, uno podría asignar ese characterem en el ciclo de 19 años y luego asignarlo a un año del calendario Juliano. El segundo uso del calendario es que cualquier historiador podría convertir fechas extraídas de las fuentes documentales de diferentes pueblos en años del período Juliano y así obtener cronologías relativas y absolutas para los eventos en cuestión (Grafton, 1975: 163). La escala establecida por Scaliger era completa respecto al principio físico en el cual se basaba la Creación, y prácticamente podía usarse para calcular con precisión las relaciones cronológicas entre cualquier cultura y localizar cualquier serie de eventos de manera absoluta. 
Una vez establecida la escala temporal absoluta de 7980 años por medio de un ciclo político y dos astronómicos, Scaliger solamente tenía que encontrar una fecha (o punto) real que sirviera como characterem, y por medio del cuál pudiera sincronizar las distintas cronologías documentales. Ese punto real fue el nacimiento de Cristo. Para establecer el año preciso, Scaliger tomó como fuente documental a Dionysius Exiguus quién había establecido el nacimiento de Cristo en el primer año del ciclo lunar, el noveno del ciclo solar y el tercero de la indicación. Por lo tanto, la operación aritmética fue establecida de la siguiente manera:

Scaliger began by establishing in what year in the 532-year Dionysian cycle Christ had been born ... The year which is the first year of the lunar cycle and the ninth of the solar cycle is 457 . (Arithmetically, 457 is the smallest number which is divisible by 19 if 1 is subtracted and also by 28 if 9 is subtracted) ... Now Scaliger had to factor in the year of the indiction. The year 457 is the seventh year of the indiction ( 457 divided by 15 leaves a remainder of 7 . The quotient is irrelevant since the indictions are not numbered consecutively) To bring the remainder around to 3 , the year of the indiction in which Dionysius put the birth of Christ, Scaliger would have to add 11, so he needed to find a number which, multiplied by 532 and divided by 15 , would leave 11 as a remainder. The answer is 8; 8 times 532 equals 4,256, plus 457 from the original cycle equals 4,713 , which is the year of Christ's birth in the Julian period (Wilcox, 1987: 199).

\section{Limites del calendario Juliano de Scaliger}

En De emendatione temporum, Scaliger fechó eventos importantes, desde la Creación hasta Constantino utilizando la datación documental y asignando una fecha en el calendario Juliano. En los últimos libros reunió tablas cronológicas para integrar todos los calendarios y épocas conocidas, algunas de estas tablas sólo conte- 
nían colecciones de números sin eventos. En 1606 publicó Thesaurus temporum, donde buscó reconstruir la crónica de Eusebio. Uno de los problemas que tuvo que enfrentar fue la integración de una cronología dinástica egipcia escrita por Manetho en el siglo III a. C. que precedió por 1, 336 años a la Creación y sobrepasó los límites de su calendario Juliano. Scaliger tuvo que resolver esta dificultad añadiendo un segundo ciclo de 7980 ańos al periodo original, el cual llamó periodo Juliano proléptico (vid. Wilcox, 1987: 201). La dificultad real, dice Wilcox, es la idea de que existen eventos anteriores a la Creación. Dos soluciones potenciales se probaron, la primera de ellas fue reorganizando los eventos de tal forma que pudiera producir una fecha más temprana para la Creación, o bien asumiendo que algunas de las dinastías de la lista de Manetho eran simultáneas a los primeros eventos bíblicos.

Para Scaliger, el ciclo de 7980 años proporcionó números que se podían sincronizar con varios calendarios porque tenían una relación real con los eventos. Para explicar el ciclo proléptico menciona que "Varro established three different times: hidden, mythic, and historical. We tend to equate the first two, even though they differ. For many times are shown to be hidden, which are not mythic; and many mythic events happened in historical time" (Scaliger apud. Wilcox, 1987: 201-202). Como expresa Donald Wilcox, el periodo Juliano continuó con la tendencia de integrar cronologías completas que comenzaron en la época helénica hasta el presente, la cronología de Scaliger tenía la capacidad de incluir toda la historia y de fechar los eventos de forma absoluta. Los eventos que cayeran fuera del periodo de 7980 simplemente se expresaban dentro de otro sistema de fechamiento.

Algo que resaltan Wilcox y Grafton es el hecho de que el sistema de Scaliger es artificial en el sentido de que la escala de 7980 ańos es independiente de los eventos que puedan ser introducidos en ella. Sin embargo, algo que no mencionan es que Scaliger, por 
primera vez en la historia de los estudios cronológicos, trata de separar las características intrínsecas al sistema de fechamiento absoluto como algo ontológicamente independiente de las características de la escala epistémica. Es decir, al buscar una fecha real que funcione como punto fijo para la sincronización de documentos históricos, Scaliger distingue la apariencia observable y mensurable de los intervalos de tiempo dentro de los sistemas cronológicos de los intervalos naturales de los eventos y procesos propiamente dichos. Por supuesto, al separar la historia de la cronología, los intervalos de tiempo naturales son los ciclos astronómicos y menciona que estos "intervals of time are like the intervals of space; just as spatial measurement can be combined, so can temporal measurement" (Scaliger apud. Wilcox, 1987: 200). La noción de fecha real está vinculada por primera vez a la noción de espacio-tiempo.

De lo anterior quiero destacar que la medición del tiempo en las cronologías antes de Scaliger arrojó resultados muy diferentes porque los valores cruzados utilizados en las sincronizaciones no eran puntos fijos reales, eran fechas convergentes registradas en distintos sistemas cronológicos. Más allá de que el nacimiento de Cristo haya sido una decisión ideológica dado que pudo escoger cualquier otro punto, el procedimiento para encontrar el punto fijo real establece de manera indirecta un valor calculado como un valor real. Guillaumin menciona que el establecimiento de un valor obtenido por medio de una medición científica depende en gran parte de que el procedimiento mediante el cual se llegó a dicho valor justifique claramente no sólo por qué ese valor es verdadero, sino sobre todo que justifique satisfactoriamente por qué los anteriores valores son falsos. Eso fue lo que sucedió. En la cronología de Scaliger las fechas que tradicionalmente habían servido como valores cruzados fueron corregidas mediante el uso del characterem. Por ejemplo, la victoria de Alejandro Magno sobre el Imperio Persa quedó fijada en la nueva cronología en el año 331 
a. C. Scaliger mostró que el rey de Babilonia Nabonasar no era la misma persona que el Salmansar bíblico y, al hacerlo, cambió la cronología bíblica (Grafton, 1991: 129).

4004 años a. C. en la cronología de Ussher

El obispo Ussher tiene cierta fama entre los estudiosos de la historia temprana de la geología, arqueología y la biología evolutiva debido a que en el siglo xvir calculó el momento exacto cuando Dios creó la Tierra. La controversia sobre el origen bíblico del mundo y la teoría de la evolución ha convertido a Ussher en un símbolo de religiosidad estrecha y oposición al progreso científico; sin embargo su trabajo refleja las tendencias científicas de su época (vid. Wilcox, 1987: 187). La importancia de Ussher, en palabras de Gould, es que representaba la más fina erudición de su tiempo. Formaba parte de una sólida tradición de investigadores, una gran comunidad de intelectuales que perseguían un fin común con una metodología aceptada (vid. Gould, 1987). Martin J. Rudwick (2014) menciona que su cronología fue una de las mejores prácticas académicas de su tiempo encargadas de construir una línea de tiempo detallada y precisa de la historia mundial.

Ussher estableció como fecha de la Creación el domingo 23 de octubre del año 4004 a.C. El cálculo se basó en principio en una tradición filológica que se remonta a la Edad Media y que establecía un intervalo de 4000 años entre La Creación y la venida de Cristo. Otra referencia para Ussher fue el cálculo de Dionysus Exiguus quién había establecido que Jesús nació 753 años después de la fundación de Roma. En la cronología de Scaliger se sabía que Herodes había muerto en el año 4 a. C., y como en el libro de Mateo registra que Herodes estaba vivo cuando Jesús nació, Ussher estableció la fecha de la Creación en 4004 a. C. Además de esto, usó por primera vez en la cronología el cálculo basado en la 
construcción del Templo de Salomón. Según el libro de Reyes I, la construcción del templo comenzó en el año 480 después del éxodo del pueblo de Israel de Egipto, y terminó de construirse en el cuarto año del reinado de Salomón. Según los cálculos de Ussher, el templo comenzó en el año 2993 A. M. (anno mundi, es decir, en el año 2993 desde la creación del mundo) y terminó de ser en el 3000 A. M., por lo cuál hay un intervalo de 1000 años más hasta la venida de Cristo.

Ussher trabajó casi en su totalidad a partir de fechas exactas (characterem), y en lo que concierne al material bíblico, igual que sus predecesores, sabía que la Biblia contenía fechas equívocas lo cual obligó a Ussher a tomar el trabajo de Scaliger:

Historically, various countries have used different methods of calculating time and years. It is necessary that some common and known standard be used to which these may be reconciled. The Julian years and months are most suitable to the common collation of times. These start on midnight, January 1, AD. Using three cycles, every year is uniquely identified. These cycles are:

a) the Roman indiction [indictio] 1 of fifteen years,

b) the cycle of the moon, 2 or golden number of nineteen years

c) the solar cycle 3 (the index of Sunday or Pascal days) of twenty-eight years (Ussher, 2003: 10).

Un problema fundamental en el cálculo bíblico fue que no había ningún evento en las cronologías del Nuevo Testamento que indicara la distancia temporal respecto a las cronologías del Antiguo. El gran mérito de Ussher fue el uso de documentos no bíblicos para establecer un punto fijo que sirvió para la unión y sincronización ambas cronologías; la fecha que podía "remendar" ambas cronologías fue la muerte de Nabucodonosor y su sucesión al trono de su hijo Amel-marduk. De acuerdo con la tradición bíblica caldea, 
la misma sobre la que trabajo Josephus, la muerte de Nabucodonosor tuvo lugar en el año 563 a.C., año que coincide con exilio de Joaquín narrado en el libro de Reyes. Este sincronismo ofrecía un punto de referencia por medio del cual fue posible enlazar ambas partes de las cronologías, específicamente con los tiempos de Salomón.

I have noted the continual passing of these years as recorded in the Bible. Hence the end of Nebuchadnezzar's reign and the beginning of his son Evilmerodach's reign was in the 3442 and year of the world (3442 AM). By collation of Chaldean history and the astronomical cannon it was in the 185th year of Nabonassar. This was 562 BC or 4152 JP (Julian Period). From this I deduced that the creation of the world happened in the beginning of the autumn of $710 \mathrm{JP}$ or $4004 \mathrm{BC}$. Using astronomical tables, I determined the first Sunday after the autumnal equinox for the year 710 JP or 4004 BC was October 23 of that year (Ussher, 2003: 11).

Este procedimiento permitió a Ussher establecer fechas calendáricas como la expulsión de Adán y Eva del Paraíso (el lunes 10 de noviembre de 4004 a. C.) o la fecha del Diluvio Universal (el miércoles 5 de mayo del 2348 a. C.). Siguiendo la cartas astronómicas más precisas de su tiempo, las Tablas Rudolfinas de Johannes Kepler, fue como Ussher identificó la Edad de la Creación.

\section{Análisis}

En los puntos anteriores expuse cuáles fueron las dificultades conceptuales, epistémicas, metodológicas y cognitivas a las que se enfrentaron los cronólogos en su intento por establecer una escala temporal que les permitiera fechar de manera absoluta toda la historia humana incluyendo el origen del Mundo. Para una com- 
prensión filosófica más completa, en este apartado discuto algunas cuestiones adicionales.

En términos de Chang y de Guillaumin cada nuevo desarrollo de los estándares de precisión en el acto de medir es imperativo para el progreso del conocimiento métrico. Chang menciona que "Progress can mean any number of things, but when it comes to the improvement of standards there are a few obvious aspects we desire: the consistency of judgments reached by means of the standard under consideration, the precision and confidence with which the judgments can be made, and the scope of the phenomena to which the standard can be applied. [...] Progress comes to mean a spiral of self-improvement if it is achieved while observing the principle of respect" (Chang, 2004: 44). Podríamos considerar la idea de auto-corrección, o de iteración epistémica como la llama Chang, como un proceso en el que las etapas sucesivas de conocimiento se crean a partir de la corrección de normas epistémicas anteriores, cuya finalidad es potencializar el logro de ciertos objetivos epistémicos. En este caso, la elaboración de una escala epistémica absoluta basada en evidencia documental y astronómica mostró que en la medición del tiempo existe un proceso que involucra el desarrollo, la consolidación y el mejoramiento de aspectos conceptuales, instrumentales y matemáticos.

Desde la antigüedad griega se conoce que las fuentes documentales contenían registros de eventos con fechas inconsistentes. Esto no llevó a rechazar la evidencia documental categóricamente, principalmente porque muchos de los sistemas cronológicos basados en documentos fueron aceptados como verdaderos por el principio de autoridad. El papel que jugó este principio epistemológico fue determinante, pues justificó el principio físico que explicaba el origen del Mundo y a partir de ahí se establecieron los límites históricos de lo humano y lo natural. El grado de confianza en el Antiguo Testamento fue tan alto que, aparte de funcionar como un 
medio de registro para transmitir datos a través de largos periodos de tiempo, proporcionó una lista de sucesiones genealógicas que internamente fueron la base para la elaboración de una primera escala ordinal. Esta lista de sucesiones genealógicas es importante epistemológicamente hablando porque nos permite ver que en la asignación de valores no existe una independencia metodológica entre los parámetros de la medición y los valores asignados; en otras palabras, hay una asignación de valores mediante la idea de quién engendró a quién como ya se mencionó.

En el caso de los primeros estudios comparativos entre el Antiguo Testamento y fuentes no bíblicas encontramos un escenario intelectual cognitivamente más enriquecedor. Si bien la primera norma fue aceptar por autoridad los límites y las fechas más tempranas de la historia del Mundo, el acuerdo básico entre los primeros cronólogos bíblicos fue producir un tipo de escala cardinal cuyos valores indicaran un orden definido de eventos a través de fechas metodológicamente bien establecidas y epistemológicamente confiables. Para calcular dichos valores se tomaron como puntos de referencia aquellos fenómenos históricos convergentes registrados en distintas fuentes documentales cuyos valores fueron calculados sobre la base los calendarios civiles y astronómicos. El grado de coincidencia entre los valores cruzados proporcionó la idea de que algunas fechas podrían ser establecidas con seguridad por medio del grado de convergencia. La cuestión epistemológica central es que la convergencia fue necesaria como un estándar epistemológico previo a la comparación entre distintos sistemas cronológicos ya que permite establecer el orden temporal de los eventos dentro de un mismo sistema cronológico y calcular los valores existentes entre ellos.

Si bien Guillaumin y Chang han señalado que el grado de convergencia entre valores calculados epistémicamente no ofrece suficientes razones para creer que dichos valores sean correctos -ya 
que estos pueden ser producto de errores sistemáticos-, el establecimiento de patrones de convergencia han sido fundamentales en las primeras etapas de las prácticas de medición ya que "proporciona buenas razones para juzgar que hemos llegado a establecer valores respaldados por evidencia provista por los datos" (vid. Guillaumin, 2016: 254-255). De acuerdo con estos autores, en los actos de medir existe un patrón en el cual una metodología usada puede que esté generando convergencia a favor de datos falsos. Sin embargo, la convergencia adquiere mayor grado de confianza cuando diferentes métodos de medición convergen hacia un mismo valor. Este mismo patrón está presente en las subsecuentes normas epistémicas. La sincronización y la tabulación fueron determinantes en el establecimiento de nuevos criterios de precisión y de justificación epistémica ya que a través de ellos se fomentó la comparación de líneas paralelas de datos entre distintos sistemas cronológicos. Como resultado, algunos eventos registrados en distintos sistemas cronológicos, o aquellos eventos simultáneos, proporcionaron cantidades significativas que permitieron establecer valores convergentes por primera vez dentro de una escala cardinal, lo cual permitió calibrar y corregir algunas fechas inconsistentes en otros sistemas de computo cronológico. Con la aplicación de técnicas filológicas para darle legitimidad a las fuentes, y con la aplicación de modelos astronómicos más precisos, no sólo se pudo identificar los errores más comunes dentro de los cálculos, sino también establecer escalas epistémicas más robustas. Más robustas porque algunas líneas paralelas de datos indicaron un mayor grado de convergencia, más constante e invariante.

Finalmente quiero discutir en qué medida ciertos fenómenos fijan realmente eventos temporales. He mencionado que el principio por autoridad fue la primera norma epistémica que establece valores a la escala ordinal seguida por la norma basada en la convergencia de fenómenos históricos. En la primera, la validez 
de la escala ordinal es asumida por autoridad ya que no hay otro punto de partida plausible para obtener conocimiento empírico. Sin embargo, un sistema cronológico basado en genealogías no podría proporcionarnos fechas absolutas, restrictivamente lo único que podría proporcionarnos es un orden temporal en términos de antes o después de. En la segunda, las escalas cardinales basadas en la convergencia, aparte de mejorar el establecimiento de los valores, permiten un ordenamiento más seguro y consistente de los fenómenos históricos; por medio de la sincronización y tabulación, por primera vez, las operaciones aritméticas se aplicaron de manera significativa para calcular algunas fechas relativamente precisas, lo que fue un paso importante para establecer los primeros puntos fijos.

Una vez constituido el sistema A. M. (anno mundi) como cronología predominante fue posible establecer algunos puntos fijos por medio de fenómenos históricos. Estos puntos, y la distancia temporal entre ellos, permitieron establecer valores mucho más confiables. Sin embargo, no perdamos de vista que fue a finales del siglo XVI cuando la cronología se convirtió en una disciplina universitaria y ello llevó a la integración de técnicas filológicas mucho más rigurosas que permitieron descartar documentos que contenían errores, o que simplemente no eran legítimos; y la integración del conocimiento astronómico basado en modelo heliocéntrico que permitió rehacer un ajuste en los calendarios astronómicos y civiles. A pesar de que el punto fijo establecido en el sistema Juliano fue arbitrario, éste permitió establecer por primera vez fechamientos absolutos de todos aquellos eventos registrados en documentos, incluyendo el momento de la Creación. La razón por la cuál prevaleció este sistema de fechamiento durante toda la tradición de cronología bíblica fue porque no había en occidente -hasta el siglo XIX- ninguna alternativa naturalizada plausible. Al no contar con algún tipo de evidencia física en contra, el grado 
de confianza en las cronologías bíblicas fue tan alto que permitió fechar cualquier evento de la historia humana conocido.

\section{Consideraciones finales}

Los problemas tratados aquí se refirieron a la medición del tiempo histórico en la tradición de cronologías bíblicas, sobre cuáles fueron las herramientas utilizadas para medirlo, y bajo qué criterios epistémicos y metodológicos se fueron desarrollando y definiendo las escalas de tiempo absolutas. Por lo tanto, las preguntas filosóficas esbozadas aquí fueron acerca del rol, la representación, la evaluación y las limitaciones epistémicas de las escalas de tiempo.

Esta consideración me permite regresar al inicio. Algunos historiadores y filósofos han mostrado equivocadamente que el nacimiento de la ciencia moderna fue una ruptura radical con las creencias pre-científicas. Sin embargo, este tipo de posturas no enseńa que el rompimiento fue un proceso largo y complejo que transformó no sólo las creencias tradicionales que se tenían acerca de la naturaleza, sino también los elementos habituales con los que se producía conocimiento histórico. Claro, ahora sabemos que el principio físico que explicaba el origen del Mundo es falso, sin embargo, el desarrollo de los artilugios utilizados en las cronologías bíblicas -no todos, pero sí algunos- siguen siendo de gran utilidad en disciplinas como la historiografía y la arqueología. El punto aquí no es que tales artilugios hayan sido correctos respecto al conocimiento que tenemos sobre el origen del mundo, sino que fueron concebidos como métodos y estándares epistemológicos adecuados y correctos para la tradición de cronólogos bíblicos. 


\section{Bibliografía}

Barr, James, 1984, "Why the World Was Created in 4004 B.C.: Archbishop Ussher and Biblical Chronology", en A lecture delivered in the John Rylands University Library of Manchester. Disponible en: https:/www.escholar.manchester.ac.uk/api/ datastream? publicationPid=uk-ac-manscw: $1 \mathrm{~m} 1647 \&$ datastreamId=POST-PEER-REVIEW-PUBLISHERS-DOCUMENT.PDF

, 1999, "Pre-Scientific Chronology: The Bible and the Origin of the World", Proceedings of the American Philosophical Society, vol. 143, núm. 3, American Philosophical Society.

Blake, Warren E., 1940, “Joseph Justus Scaliger”, The Classical Journal 36, núm. 2, pp. 83-91.

Buchwald, Jed y Mordechai Feingold, 2013, Newton and the Origin of Civilization, Princeton University Press, Nueva York.

Cesarea, Eusebio de, 2008, Chronicle, Robert Bedrosian (trad.), 2008. Disponible en: http://www.tertullian.org/fathers/eusebius_chronicon_02_text.htm

Chang, Hasok, 2004, Inventing Temperature: Measurement and Scientific Progress, Oxford Studies in Philosophy of Science, Oxford University Press, Oxford.

De Jonge, H. J., 1996, "Joseph Scaliger's Historical Criticism of the New Testament", Novum Testamentum 38, núm. 2, pp. 176-93.

Feldman, L. H., 1996, Jew and Gentile in the Ancient World. Attitudes and Interactions from Alexander to Justinian, Princeton University Press, Nueva York.

Grafton, Anthony, 1983, Joseph Scaliger: A Study in the History of Classical Scholarship, vols. 1 y II, Textual Criticism and Exegesis, Oxford University Press, Oxford. 
, 1975, "Joseph Scaliger and Historical Chronology: The Rise and Fall of a Discipline", History and Theory, vol. 14, núm. 2, Mayo, pp. 156-185.

Grafton, Anthony y Megan Williams, 2006, Christianity And The Transformation Of The Book: Origen, Eusebius, And The Library Of Caesarea, Harvard University Press, Cambridge.

Gould, Stephen J., 1987, Time's Arrow. Time’s Cycle, Harvard University Press, Cambridge.

, 2011, The Lying Stones Of Marrakech: Penultimate Reections In Natural History. Harvard University Press, Cambridge.

Guillaumin, Godfrey, 2012, "De las cualidades a las magnitudes: la integración cognitiva de la medición en el surgimiento de la astronomía moderna”, Signos Filosóficos, UAM. México.

,2016, Génesis de la medición celeste. Una historia cognitiva del crecimiento de la medición científica, UAM-I, México.

Kitcher, Philip, 2011, "Epistemology Without History is Blind", Erkenntnis, vol. 3, núm. 75, pp. 505-524.

Kuhn, Thomas S., 1977, The essential tension, University of Chicago Press, Chicago.

, 1996, The structure of scientific revolutions, University of Chicago Press, Chicago.

Lesne, Annick, 2017, "Time Variable and Time Scales in Natural Systems and Their Modeling", en Time of Nature and the $\mathrm{Na}$ ture of Time. Philosophical Perspectives of Time in Natural Sciences, Christophe Bouton y Philippe Huneman (eds.), Boston Studies in the Philosophy and History of Science, Springer.

Momigliano, Arnaldo, 1978, "Greek Historiography", en History and Theory. Disponible en: http://www.jstor.org/ stable/2504899

Nothaft, C., 2011, "Noah's Calendar: The Chronology Of The Flood Narrative And The History Of Astronomy In Six- 
teenth-And Seventeenth-Century Scholarship", Journal of the Warburg and Courtauld Institutes, núm. 74, pp. 191-211.

Piggott, Stuart, 1959. Approach to Archaeology. Adam \& Charles Black, London.

Rajak, Tessa, 2003, Josephus. The Historian and His Society, Gerald Duckworth \& Co, Londres.

Richet, Pascal, 2007, A Natural History of Time, The University of Chicago Press, Chicago.

Rudwick, Martin J. S., 2014, Earth's deep history : how it was discovered and why it matters. The University of Chicago Press, Chicago.

Schneer, Cecil, 1952, "The Rise of Historical Geology in the Seventeenth Century", Isis, vol. 45, núm. 3, The University of Chicago Press.

Syncellus, Georgius, 1829, Chronographia, Oxford University.

Ussher, James, 1650, Annales veteris testamenti, a prima mundi origine deducti. Disponible en: https://archive.org/details/AnnalsOfTheWorld

Wallraff, Martin, 2007, Iulius Africanus Chronographiae. The Extant Fragments, Walter de Gruyter, Berlín/Nueva York.

Weintraub, David A., 2012, La edad del universo, Editorial Crítica, Barcelona.

Wilcox, Donald, 1987, The Measure of Times Past. Pre-Newtonian Chronologies And The Rhetoric Of Relative Time, The University of Chicago Press, Chicago. 